07

\title{
Роль энергии фотонов возбуждения в динамике фотоиндуцированных носителей заряда в сверхрешеточных гетероструктурах InGaAs/InAIAs
}

\author{
(C) А.М. Буряков, ${ }^{1}$ Д.И. Хусяинов, ${ }^{1,}$ Е.Д. Мишина, ${ }^{1}$ \\ Р.А. Хабибуллин, ${ }^{2,3}$ А.Э. Ячменев, ${ }^{2,3}$ Д.С. Пономарев ${ }^{2,3}$ \\ ${ }^{1}$ МИРЭА - Российский технологический университет „РТУ МИРЭА“, \\ Москва, Россия \\ ${ }^{2}$ Институт сверхвысокочастотной полупроводниковой электроники РАН, \\ Москва, Россия \\ ${ }^{3}$ Институт общей фризики им. А.М. Прохорова РАН, Москва, Россия \\ ฯ E-mail: Dinar1434429@mail.ru
}

Поступило в Редакцию 24 июля 2018 г.

Исследовано влияние энергии фотонов возбуждения на времена релаксации фотовозбужденных носителей заряда. Проведены оценка возникающих механизмов релаксации и анализ коэффициента отражения в сверхрешеточных гетероструктурах InGaAs/InAlAs. Построена модель изменения коэффициента отражения в барьерном слое InAlAs в зависимости от энергии фотонов возбуждения. Объяснено резонансное поведение коэффициента отражения.

DOI: $10.21883 /$ PJTF.2018.23.47022.17469

Изучение сверхбыстрой динамики фотовозбужденных носителей заряда (ФНЗ) в полупроводниках является одной из ключевых задач для определения характеристик фотопроводящих антенн (ФПА) на их основе. Исследования фотопроводящих структур InGaAs/InP показали наличие двухфотонного поглощения (two-photon absorption) и поглощения излучения на свободных носителях (free-carrier absorption), влияние которых на динамику фотовозбужденных носителей заряда приводит к дополнительной экспоненциальной зависимости, обусловленной рассеянием носителей заряда на фононах [1]. Динамические свойства ФНЗ, связанные с процессами рассеяния носителей на фононах, процессами, соответствующими режиму горячих носителей заряда, и изменением 
коэффициента отражения в зависимости от энергии фотонов возбуждения, слабо изучены в гетероструктурах и представляют особый интерес. Время жизни в подобных структурах было тщательно изучено в работе [2] в зависимости от концентрации легирования бериллием, тогда как исследование времени жизни носителей заряда в зависимости от энергии фотонов лазерной накачки является новой и перспективной задачей. Проведение таких исследований также обусловлено необходимостью определения наиболее эффективного диапазона работы ФПА на основе сверхрешеточных гетероструктур InGaAs/InAlAs.

Исследование динамики носителей заряда проводилось в образце на основе сверхрешетки, состоящей из 30 периодов последовательных слоев $\mathrm{In}_{0.62} \mathrm{Ga}_{0.38} \mathrm{As} / \mathrm{In}_{0.57} \mathrm{Al}_{0.43} \mathrm{As}$ с толщинами 12 и $4 \mathrm{~nm}$ соответственно. Низкая температура роста $T_{s}=400^{\circ} \mathrm{C}$ слоя $\operatorname{In}_{0.57} \mathrm{Al}_{0.43} \mathrm{As}$ обеспечивает большое число ловушек для носителей заряда. При туннелировании между фотопроводящими слоями $\mathrm{In}_{0.62} \mathrm{Ga}_{0.38} \mathrm{As}$ электроны захватываются ловушками в $\operatorname{In}_{0.57} \mathrm{Al}_{0.43} \mathrm{As}$, которые служат центрами рекомбинации; их наличие приводит к увеличению сопротивления всей структуры. Исследуемый образец создан на основе работ авторов [3-5] по изучению генерации терагерцевого $(\mathrm{THz})$ излучения в таких полупроводниковых структурах.

Кристаллическая структура сверхрешетки InGaAs/InAlAs была аттестована методом рентгеновской дифрактометрии. На основе результатов работы [6] были определены значения ширины запрещенной зоны в долинах $\Gamma, X, L$ зоны проводимости в твердых растворах InGaAs и InAlAs. Для $\operatorname{In}_{x} \mathrm{Ga}_{1-x}$ As c мольной долей $x=0.62$ параметры зонной структуры составили $E_{\Gamma}=0.64 \mathrm{eV}, E_{X}=1.24 \mathrm{eV}, E_{L}=1.23 \mathrm{eV}$. Для $\mathrm{In}_{y} \mathrm{Al}_{1-y} \mathrm{As}$ с мольной долей $y=0.57 E_{\Gamma}=1.34 \mathrm{eV}, E_{X}=1.72 \mathrm{eV}$, $E_{L}=1.63 \mathrm{eV}$.

Исследование временно́й динамики ФНЗ в сверхрешетке InGaAs/InAlAs при энергиях оптического возбуждения $1.23-1.65 \mathrm{eV}$ проводилось методом оптического „возбуждения-зондирования“ с использованием твердотельного лазера на основе кристалла сапфира, легированного ионами титана, с длительностью импульса $100 \mathrm{fs}$ и частотой следования импульсов $80 \mathrm{MHz}$. Подробное описание экспериментальной установки приведено в работе [7]. Спектральные характеристики были измерены при перестройке длины волны оптической накачки и зондирования от 750 до $1000 \mathrm{~nm}$.

10* Письма в ЖТФ, 2018, том 44, вып. 23 


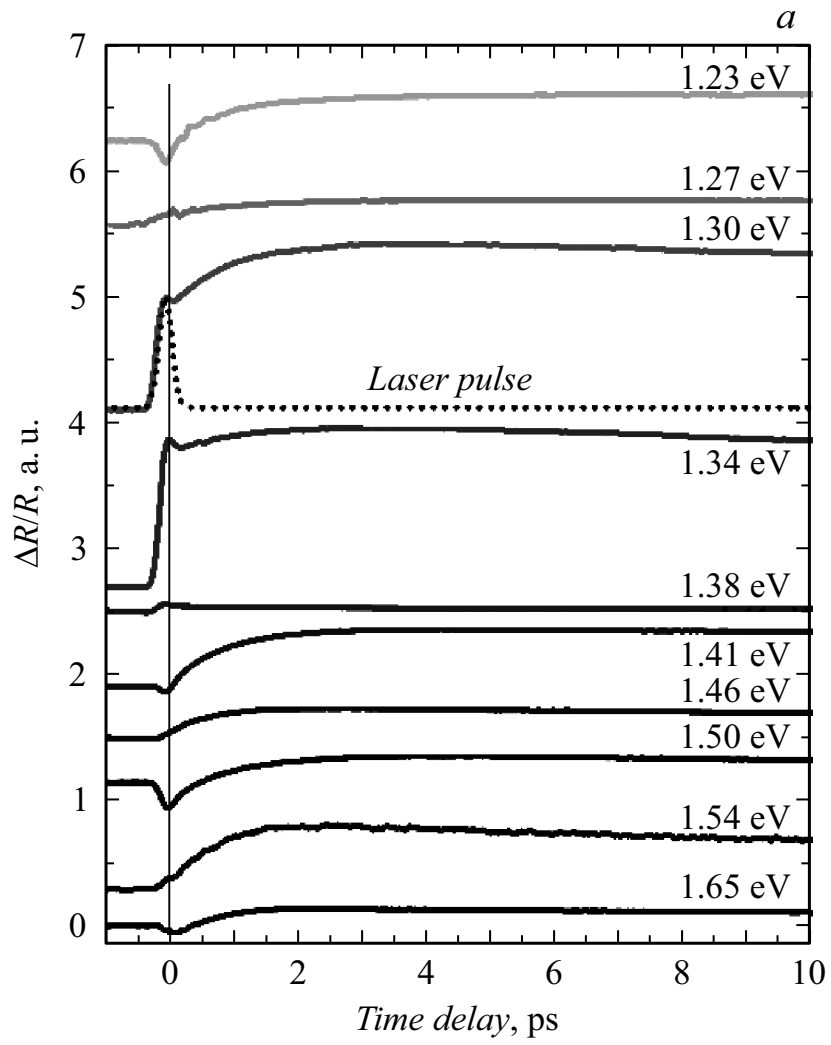

Рис. 1. Зависимости изменения коэффициента отражения от времени задержки между импульсами накачки и зондирования. Представлен спектральный набор кривых для энергий оптической накачки $1.23-1.65 \mathrm{eV}$. $a$ - короткий диапазон, $b$ - длинный диапазон. Для наглядности зависимости сдвинуты относительно друг друга по оси $\Delta R / R$.

На рис. 1, $a$ и $b$ представлены экспериментальные зависимости в диапазоне до 10 и 100 ps с шагом в 0.04 и 1 ps соответственно. Вариация диапазонов обоснована максимальным временны́м разрешением, которое позволяет оценить время рассеяния носителей заряда на фононах и процессов, соответствующих нетермическому режиму 


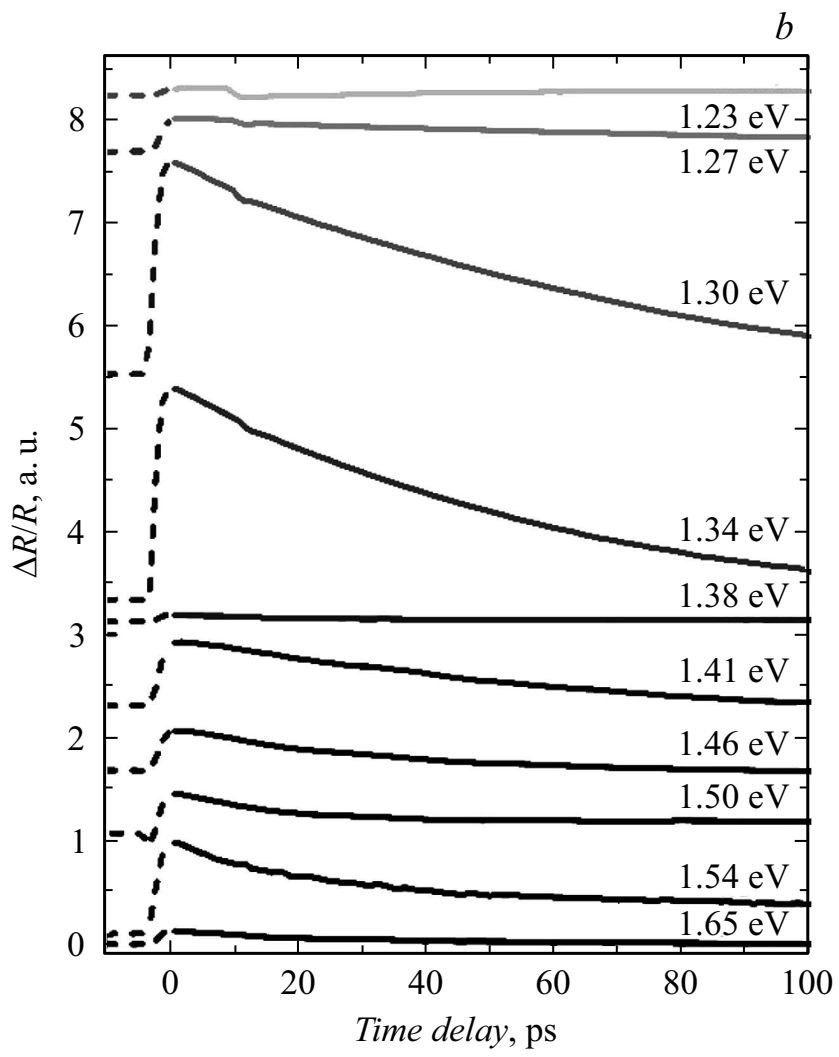

Рис. 1 (продолжение).

( $<2 \mathrm{ps})$, режиму горячих носителей $(\sim 1-100 \mathrm{ps})$ и изотермическому режиму (>100 ps) [8]. В соответствии с режимами, перечисленными выше, динамику ФНЗ можно разделить на пять процессов: момент возбуждения, обусловленный пришедшим лазерным импульсом $(\sim 100 \mathrm{fs})$, термализация носителей заряда $(\sim 150 \mathrm{fs})$, рассеяние носителей заряда на фононах с характерным временем $\tau_{1}$, процесс, связанный с перераспределением энергии, зависящей от скорости охлаждения носителей заряда до температуры кристаллической решетки, с временем $\tau_{2}$ и, наконец, процесс перехода всех носителей в равновесное состояние с

Письма в ЖТФ, 2018, том 44, вып. 23 
временем $\tau_{3}$. Следует отметить, что в некоторых случаях $\tau_{2}$ представляет собой сумму двух нелинейных процессов $[8]$.

На рис. 1, $a$ момент возбуждения неравновесных носителей хорошо виден и сопоставим во временно́м масштабе с длительностью лазерного импульса. Значение коэффициента отражения в момент воздействия фемтосекундного лазерного импульса принимает отрицательные и положительные значения. Согласно данным [9], такое поведение коэффициента отражения в момент возбуждения обусловлено нелинейностью показателя преломления вблизи энергии, соответствующей ширине запрещенной зоны в полупроводниках III-V групп.

Для качественной оценки времени жизни $Ф \mathrm{H}$, за которое происходят процессы с $\tau_{1}, \tau_{2}$ и $\tau_{3}$, использвалась феноменологическая функция экспонент, описывающая изменение коэффициента отражения $\Delta R / R$ :

$$
\frac{\Delta R}{R}\left(t_{d}\right)=\sum_{i}^{3} \frac{A_{i}}{2} \exp \left(\frac{w^{2}}{4 \tau_{i}^{2}}-\frac{\tau_{d}}{\tau_{i}}\right)\left[\operatorname{Erf}\left(\frac{\tau_{d}}{w}-\frac{w}{2 \tau_{i}}\right)+1\right],
$$

где $i$ - номер процесса релаксации, $\tau_{i}$ - время, отвечающее за процесс релаксации, $w$ - длительность импульса, $t_{d}$ - время задержки между импульсами.

Аппроксимация проводилась на короткой (рис. $1, a)$ и длинной (рис. $1, b)$ временно́й зависимостях для повышения точности оценки $\tau_{2}$. Время $\tau_{1}$ процесса, обусловленного рассеянием носителей на фононах, в зависимости от энергии накачки менялось от 0.6 до $1.9 \mathrm{ps}$.

На рис. 2, $a$ представлена зависимость, соответствующая временно́й константе $\tau_{2}$. Константа $\tau_{2}$ является наиболее важным параметром, так как она характеризует ширину спектра THz-излучения, детектируемого антенной на основе исследуемого материала: чем меньше $\tau_{2}$, тем шире спектр детектирования ФПА [10]. Анализируя энергетические состояния слоев сверхрешетки, можно отметить, что переход энергии фотонов возбуждения выше запрещенной зоны InAlAs добавляет механизм сверхбыстрой релаксации, обусловленной наличием ловушек в этом слое. В связи с этим мы наблюдаем снижение времени жизни носителей заряда $\tau_{2}$ при высоких энергиях возбуждения. Однако значение времени жизни при энергии фотонов, равной $1.23 \mathrm{eV}$, тоже принимает низкое значение, что может быть связано с влиянием $X$-долины в InGaAs.

Зависимость коэффициента отражения от энергии фотонов оптической накачки для времен до 100 ps представлена на рис. $2, b$. Времена, 

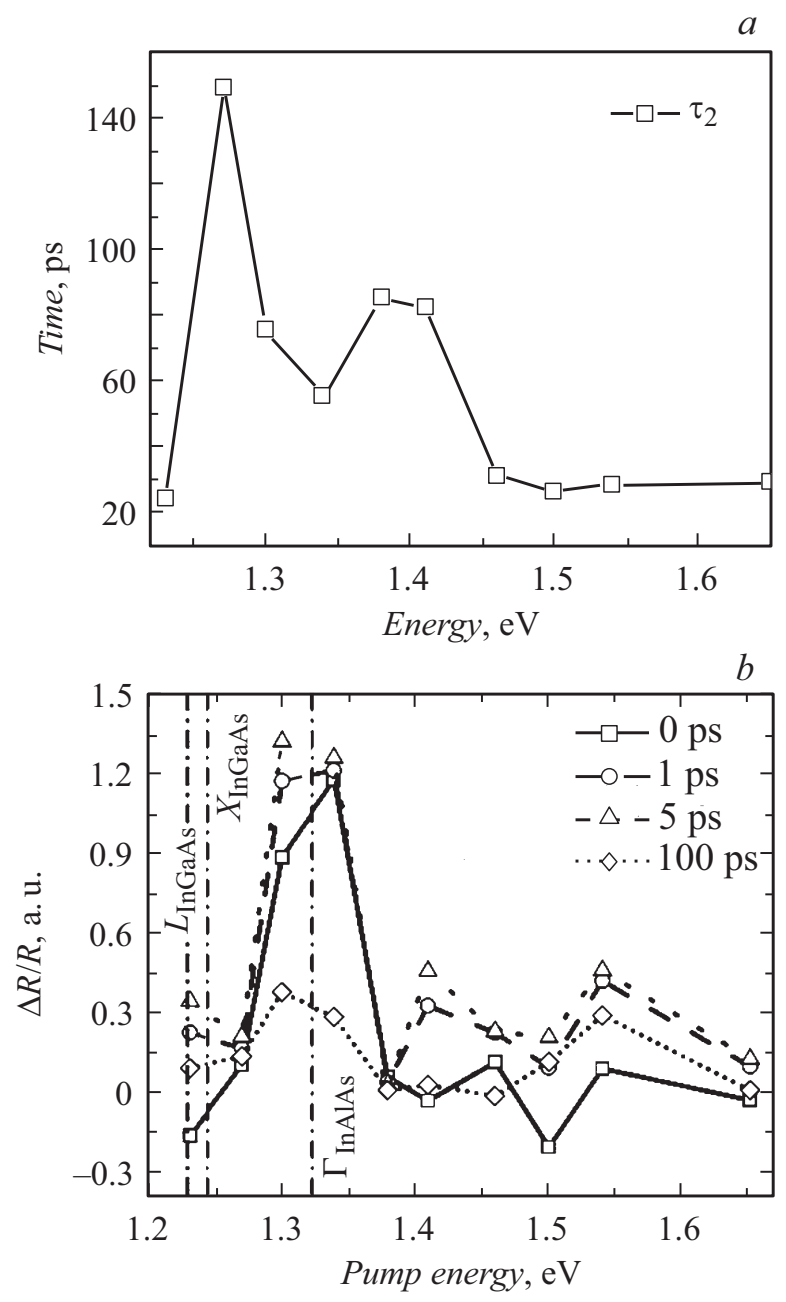

Рис. 2. $a-$ зависимости временно́й константы $\tau_{2}$ от энергии оптической накачки, $b-$ зависимость коэффициента отражения в различные моменты времени задержки от энергии оптической накачки.

Письма в ЖТФ, 2018, том 44, вып. 23 
при которых построены зависимости относительного коэффициента отражения от энергии фотонов оптической накачки $\tau=0,1,5,100 \mathrm{ps}$, были зафиксированы таким образом, чтобы охарактеризовать четыре перечисленных выше процесса. Из рис. 2, $b$ видно, что от процесса зависит лишь амплитуда кривой, а не ее характер. Самый высокий коэффициент отражения достигается при значении $1.34 \mathrm{eV}$, которое обусловлено величиной энергии запрещенной зоны в $E_{g}=1.321 \mathrm{eV}$ в InAlAs. Со временем пик коэффициента отражения при энергии фотонов оптической накачки $1.34 \mathrm{eV}$ постепенно спадает и в момент времени, соответствующий $100 \mathrm{ps}$, перестает выделяться из общей картины динамики ФНЗ.

Для качественного описания изменения коэффициента отражения в диапазоне энергий фотонов оптического возбуждения от 1.23 до $1.65 \mathrm{eV}$ использовалась модель, представленная в работе [11]. Использование данной модели корректно для моделирования изменения коэффициента отражения в InAlAs, так как это широкозонный полупроводник и из-за низкой температуры роста в нем присутствует высокая концентрация центров безызлучательной рекомбинации.

Динамика носителей заряда учитывалась при помощи модели Шокли-Рида-Холла [10-12]:

$$
\begin{gathered}
\frac{d n}{d t_{d}}=\frac{I\left(t_{d}\right)}{h v} \alpha-C_{n} n\left(N_{t}-n_{t}\right)+n_{t} C_{n}^{t h}, \\
\frac{d n_{t}}{d n_{d}}=-C_{p} n_{t} p+C_{n} n\left(N_{t}-n_{t}\right)+C_{p}^{t h}\left(N_{t}-n_{t}\right)-C_{n}^{t h} n_{t}, \\
\frac{d p}{d t_{d}}=\frac{I\left(t_{d}\right)}{h v} \alpha-C_{p} n_{t} p+C_{p}^{t h}\left(N_{t}-n_{t}\right),
\end{gathered}
$$

где $p$ - равновесная концентрация дырок, $n-$ равновесная концентрация электронов, $n_{t}$ - концентрация неионизированных безызлучательных центров рекомбинации, $N_{t}$ - концентрация ионизированных безызлучательных центров рекомбинации, $I\left(t_{d}\right)$ - концентрация фотовозбужденных носителей заряда, инжектируемых фемтосекундным импульсом, $\alpha$ - коэффициент поглощения для исследуемого материала, $C_{p}-$ коэффициент захвата дырок, $C_{n}$ - коэффициент захвата электронов, $C_{n}^{t h}, C_{p}^{t h}-$ коэффициенты, описывающие вероятность переизлучения квазичастицы в валентную зону или зону проводимости.

Письма в ЖТФ, 2018, том 44, вып. 23 
Для моделирования изменения концентрации носителей заряда в зависимости от энергии фотонов оптического возбуждения время было зафиксировано на моменте возбуждения фемтосекундным импульсом 0 ps. Равновесная концентрация электронов была выбрана равной $10^{17} \mathrm{~cm}^{-3}$, концентрация дырок - равной $10^{18} \mathrm{~cm}^{-3}$, концентрация безызлучательных центров рекомбинации $n_{t}=10^{19} \mathrm{~cm}^{-3}$.

Зависимость коэффициента отражения от энергии фотонов оптического возбуждения определяется комплексным показателем преломления с действительной и мнимой частями, которые соотносятся при помощи уравнения Крамерса-Кронига. Для действительной и мнимой частей коэффициента преломления при высоких значениях плотности ФНЗ необходимо учесть два эффекта: заполнение зоны проводимости и перенормировку запрещенной зоны ( $\Delta \alpha_{B F}$ и $\Delta \alpha_{B G R}$ соответственно), которые записываются следующим образом [13]:

$$
\begin{gathered}
\Delta \alpha_{B F}=\sum_{i=h h, l h} \frac{C_{i}}{E} \sqrt{E-E_{g}+\Delta E_{B G R}\left(t_{d}\right)}\left[f_{v}\left(t_{d}\right)-f_{c}\left(t_{d}\right)-1\right], \\
\Delta \alpha_{B G R}=\sum_{i=h h, l h} \frac{C_{i}}{E}\left(\sqrt{E-E_{g}+\Delta E_{B G R}\left(t_{d}\right)}-\sqrt{E-E_{g}}\right),
\end{gathered}
$$

где $\Delta E_{B G R}=\gamma\left(\Delta n\left(t_{d}\right)^{1 / 3} / m_{e}+\Delta p\left(t_{d}\right)^{1 / 3} / m_{d h}\right) \quad$ - фотовозбужденная ренормализация запрещенной зоны, $m_{d h}=\left(m_{t h}^{3 / 2}+m_{h h}^{3 / 2}\right)^{2 / 3}$, значение константы $\gamma$ взято из $[11] ; f_{v}$ и $f_{c}$ представляют собой функции Ферми-Дирака для электронов и дырок в валентной зоне и зоне проводимости соответственно:

$$
\begin{gathered}
f_{c}\left(t_{d}\right)=\left(1+\exp \left(\frac{E_{a h h, a l h}-E_{f c}\left(t_{d}\right)}{k T}\right)\right)^{-1}, \\
f_{v}\left(t_{d}\right)=\left(1+\exp \left(\frac{E_{h h, l h}-E_{f v}\left(t_{d}\right)}{k T}\right)\right)^{-1},
\end{gathered}
$$

$C_{h h, l h}$ - константы, которые описывают параметры материала [13], индексы $h h, l h$ соответствуют тяжелым и легким дыркам; $E_{h h, l h}-$ энергии в валентной зоне, $E_{a h h, a l h}$ - энергии в зоне проводимости; $E_{f c}, E_{f v}-$ зависящие от носителей заряда квазиуровни Ферми для

Письма в ЖТФ, 2018, том 44, вып. 23 
электронов и дырок, которые могут быть рассчитаны с помощью уравнения Томаса-Нильсона, полученного эмпирической аппроксимацией для полупроводников при учете параболической зоны [13]:

$$
\begin{gathered}
E_{f c}\left(t_{d}\right)=k T\left(\ln \left(\frac{\Delta n\left(t_{d}\right)}{N_{c}}\right)+\frac{\Delta n\left(t_{d}\right)}{N_{c}}\left(u+v \frac{\Delta n\left(t_{d}\right)}{N_{c}}\left(u+\sqrt{\frac{\Delta n\left(t_{d}\right)}{N_{c}}}\right)\right)^{-\frac{1}{4}}\right), \\
E_{f v}\left(t_{d}\right)=k T\left(-\left\{\ln \left(\frac{\Delta p\left(t_{d}\right)}{N_{v}}\right)\right.\right. \\
\left.\left.+\frac{\Delta p\left(t_{d}\right)}{N_{v}}\left(u+v \frac{\Delta p\left(t_{d}\right)}{N_{v}}\left(u+\sqrt{\frac{\Delta p\left(t_{d}\right)}{N_{v}}}\right)\right)^{-\frac{1}{4}}\right\}-E_{g}\right)
\end{gathered}
$$

где $u=64$ и $v=55 \cdot 10^{-3}-$ феноменологические константы [13]. Коэффициент поглощения может быть записан при помощи соотношения Крамерса-Кронига [9], которое позволяет рассчитать фотовозбужденное изменение в действительной части коэффициента преломления

$$
\Delta \eta\left(\hbar \omega, t_{d}\right)=\frac{\hbar c}{\pi} \int_{e}^{+\infty} \frac{\Delta \alpha\left(E, t_{d}\right)}{E^{2}-(\hbar \omega)^{2}} d E .
$$

Фотовозбужденное изменение мнимой части коэффициента преломления записывается как

$$
\Delta \kappa\left(\hbar \omega, t_{d}\right)=\frac{c}{2 \omega} \Delta \alpha\left(\hbar \omega, t_{d}\right)
$$

Дифференциальный коэффициент отражения рассчитывается из уравнения Френеля

$$
\frac{\Delta R\left(\hbar \omega, t_{d}\right)}{R_{0}}=\frac{1}{R_{0}}\left(\Delta \eta\left(\hbar \omega, t_{d}\right) \frac{\partial R}{\partial \eta}+\Delta \kappa\left(\hbar \omega, t_{d}\right) \frac{\partial R}{\partial \kappa}\right) .
$$

Используя уравнения (5)-(10), мы провели расчет коэффициента отражения в зависимости от энергии фотонов оптического возбуждения. Результаты расчета приведены на рис. 3. При энергии фотонов $1.321 \mathrm{eV}$, соответствующей ширине запрещенной зоны $\operatorname{In}_{0.57} \mathrm{Al}_{0.43} \mathrm{As}$, наблюдается 


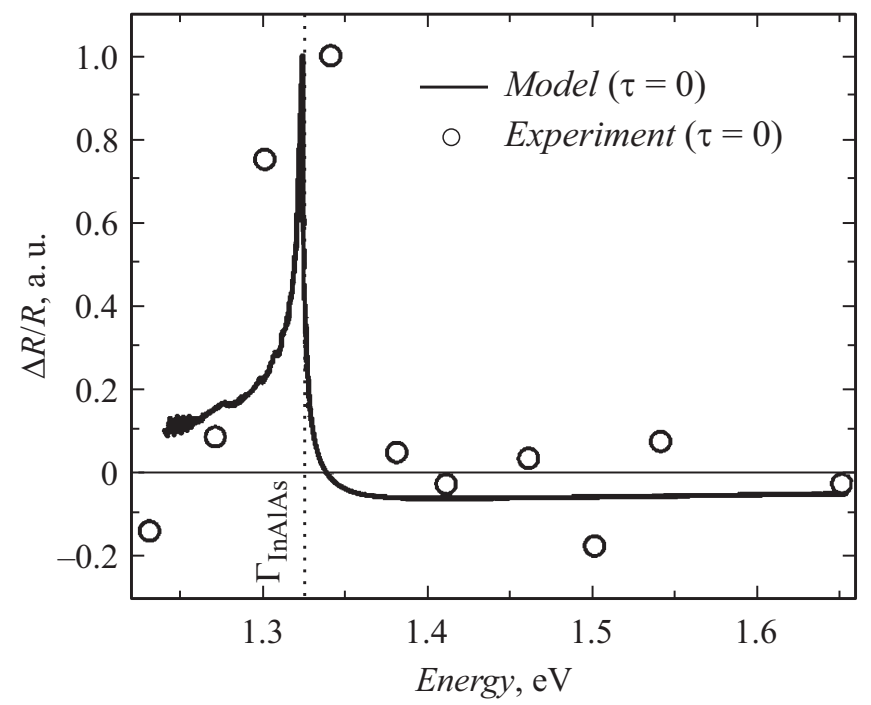

Рис. 3. Зависимость дифференциального коэиффициента отражения в момент времени $\tau=0$ от энергии оптического возбуждения в InAlAs. Точки экспериментальные данные.

интенсивный пик относительного коэффициента отражения, что согласуется с экспериментальными данными. Пик при энергиях, близких к ширине запрещенной зоны $\operatorname{In}_{0.57} \mathrm{Al}_{0.43} \mathrm{As}\left(E_{g}=1.321 \mathrm{eV}\right)$, объясняется доминированием нелинейного эффекта перенормировки запрещенной зоны в момент максимальной интенсивности лазерного импульса. При энергиях $1.46-1.65 \mathrm{eV}$ наблюдаются отрицательные значения коэффициента отражения, которые также обусловлены нелинейным эффектом перенормировки запрещенной зоны, который доминирует над эффектом заполнения зоны проводимости.

В заключение отметим, что нами была рассмотрена временна́я динамика ФН3 в сверхрешеточной гетероструктуре InGaAs/InAlAs методом оптической „накачки-зондирования“, что позволило оценить времена характерных сверхбыстрых процессов в структуре (рассеяние носителей заряда на фононах, рекомбинация и т.д.) в зависимости от энергии фотонов оптического возбуждения. Была построена модель

Письма в ЖТФ, 2018, том 44, вып. 23 
изменения коэффициента отражения в барьерном слое InAlAs, которая объясняет пик максимального значения коэффициента отражения при энергии фотонов $1.34 \mathrm{eV}$ нелинейными процессами, возникающими при энергиях фотонов оптического возбуждения, сопоставимых с энергией запрещенной зоны полупроводника. Нами было показано, что наименьшее время рекомбинации для исследуемой структуры достигается при энергии фотонов накачки $1.5 \mathrm{eV}$ и составляет $27 \mathrm{ps}$. Полученные данные свидетельствуют о перспективности использования сверхрешеточных гетероструктур InGaAs/InAlAs в качестве базового материала для широкополосного детектора THz-излучения, работающего с высокоэнергетическим лазерным возбуждением.

Работа выполнена при финансовой поддержке Министерства образования и науки РФ (государственное задание № 3.7331.2017/9.10) и РФФИ (гранты 18-02-00843 и 16-29-14029 офи_м). Эпитаксиальный рост сверхрешеточной гетероструктуры InGaAs/InAlAs проведен А.Э. Ячменевым, Д.С. Пономаревым и Р.А. Хабибуллиным при финансовой поддержке гранта РНФ 18-79-10195 и гранта Президента МК5450.2018.2. Экспериментальные исследования выполнены с использованием оборудования ЦКП „УНО „Электроника“.

\section{Список литературы}

[1] Langlois P., Joschko M., Thoen E.R., Koontz E.M., Kärtner F.X., Ippen E.P., Kolodziejski L.A. // Appl. Phys. Lett. 1999. V. 75. N 24. P. 3841-3843.

[2] Dietz R.J.B., Globisch B., Roehle H., Stanze D., Góbe T., Schell M. // Opt. Express. 2014. V. 22. N 16. P. 19411-19422.

[3] Пономарев Д.С., Хабибуллин Р.А., Ячменев А.Э., Павлов А.Ю., Слаповский Д.Н., Глинский И.А., Лаврухин Д.В., Рубан О.А., Мальцев П.П. // ФТП. 2017. Т. 51. В. 9. С. $1257-1272$.

[4] Глинский И.А., Зенченко Н.В., Мальцев П.П. // Рос. технологический журнал. 2016. Т. 4. № 3. С. 27-36.

[5] Галиев Г.Б., Пушкарёв С.С., Буряков А.М., Билык В.Р., Мишина Е.Д., Климов Е.А., Васильевский И.С., Мальцев П.П. // ФТП. 2017. Т. 51. В. 4. C. $529-534$.

[6] Vurgaftman I., Meyer J.R., Ram-Mohan L.R. // J. Appl. Phys. 2001. V. 89. N 11. P. 5815-5875. 
[7] Хусяинов Д.И., Буряков А.М., Билык В.Р., Мишина Е.Д., Пономарев Д.С., Хабибуллин Р.А., Ячменев А.Э. // Письма в ЖТФ. 2017. Т. 43. В. 22. C. $48-54$.

[8] Shah J. Ultrafast spectroscopy of semiconductors and semiconductor nanostructures. Springer Ser. in Solid-State Sciences. Berlin-Heidelberg: Springer, 1999. V. 115. 522 p.

[9] Prabhu S.S., Vengurlekar A.S. // J. Appl. Phys. 2004. V. 95. N 12. P. 7803-7812.

[10] Wells N.P., Belden P.M., Demers J.R., Lotshaw W.T. // J. Appl. Phys. 2014. V. 116. N 7. P. 73506.

[11] Ortiz V., Nagle J., Lampin J.-F., Péronne E., Alexandrou A. // J. Appl. Phys. 2007. V. 102. N 4. P. 43515.

[12] Khusyainov D.I., Dekeyser C., Buryakov A.M., Mishina E.D., Galiev G.B., Klimov E.A., Pushkarev S.S., Klochkov A.N. // Int. J. Mod. Phys. B. 2017. V. 31. N 27. P. 1750195 (1-9).

[13] Bennett B.R., Soref R.A., Del Alamo J.A. // IEEE J. Quantum Electron. 1990. V. 26. N 1. P. $113-122$. 